\title{
A Comprehensive Clinical Approach to Hypoxic Ischaemic Encephalopathy in Term Infants: A Review
}

\section{Chakradhar Maddela*}

Consultant Neonatologist, Super Speciality, Neonatology, ESIC Medical College, Sanath Nagar, Hyderabad, India

*Corresponding Author: Chakradhar Maddela, Consultant Neonatologist, Super Speciality, Neonatology, Sanath Nagar, Hyderabad, India.
Received: January 03, 2022

Published: January 31, 2022

(C) All rights are reserved by Chakradhar

Maddela.

\section{Abstract}

Introduction: Hypoxic ischemic encephalopathy (HIE) is the manifestation of multi-organ dysfunction after perinatal asphyxia. Out of 135 million live born babies in each year globally, there were 1.2million intrapartum still births, 717,000 intrapartum neonatal deaths, 1.15million cases of neonatal encephalopathy (NE), 287,000 neonatal deaths due to NE and 413,000 babies survived with neurodevelopmental impairment [1]. The incidence of HIE is 1-2 per 1000 births in rich countries and 10-20 per 1000 births [2] in low to middle income countries. Approximately ninety nine percent of HIE case related deaths are taking place in developing countries. It is a huge burden on these countries for tackling. The available trusted specific treatment, therapeutic hypothermia (HT) is less implemented and showed varied results of outcome. There was not much progress in preventing these deaths in low to middle income countries (LMIC).

Methods: An online and manual literature search was conducted in November 2021 through PubMed, Cochrane library, Google Scholar, Online Google chrome search, texts and articles related to the title topic with keywords HIE, Post-resuscitation neonatal care, term infants, management and comprehensive approach. Articles were also collected from citations and references of the searched study papers. Search restricted to English language, free full text articles, human and neonates - term and late preterm. Animal studies and studies involving preterm infants were excluded. Eighteen study articles from PubMed, 17 articles from Cochrane library and another 22 articles relevant to the title topic were collected. These articles were analysed after going through the tile and abstract sections.

Results: The collected study papers were narrated with the following subheadings - definition, pathophysiology, HIE staging, cooling criteria, cooling in High income countries (HIC), HIE and cooling in LMIC, HIE and therapeutic strategies at low resource setting (LRS), cooling HIE cases who do not fit into cooling criteria, newer potential therapeutic strategies for HIE, outcome predictors of HIE, counselling for parents, long-term health problems and conclusions.

Definition: Asphyxia is a clinical condition that occurs from inadequate gas exchange and characterized by hypoxia, hypercarbia and acidosis. Perinatal asphyxia occurs during or prior to labour. Perinatal asphyxia is failure to initiate and sustain breathing at birth (World Health Organization 1997) [3]. HIE is the multi-organ manifestation after perinatal asphyxia. "NE is a syndrome of disturbed neurological function in the earliest days of life in an infant born at or beyond 35 weeks of gestation, manifested by a subnormal level of consciousness or seizures and often accompanied by difficulty with initiating and maintaining respiration and depression of tone and reflexes." [4].

Keywords: Hypoxic Ischaemic; Encephalopathy; Term Infants; Asphyxia 
American college of obstetrics and gynaecologists 2014 (4) made the following assessment criteria while labelling a case as perinatal asphyxia:

- An Apgar score < 5 at 5 and 10 minutes

- $\quad$ Evidence of acidaemic state in foetus or new-born $(\mathrm{pH}<7$, BE $-12 \mathrm{mmol}$ or both).

- Acute brain injury in neonatal neuroimaging (Magnetic resonance imaging -MRI/cranial ultrasound scanning - cUSS/ Computerized tomography - CT Brain)

- Multi-organ dysfunction in the early life of neonate with HIE.

- A sentinel event at or around birth (Antenatal haemorrhage, cord prolapse and uterine rupture, amniotic fluid embolism, maternal cardiovascular collapse and exsanguination of foetus).

- Abnormal foetal heart rate pattern during peripartum period.

- $\quad$ Patterns of brain injury consistent with HIE on imaging.

- $\quad$ Cerebral palsy - dyskinetic, spastic quadriplegia (late manifestation).

Pathophysiology

Term infants with apnoea at birth and peripartum foetal bradycardia after a sentinel event develop primary energy failure (acute hypoxic-ischemic effect). Primary energy failure is characterised by acute loss of ATP reserves, $\mathrm{Na} / \mathrm{K}$ pump failure, $\mathrm{Na}+$ influx, cytotoxic cerebral oedema, anaerobic glycolysis with lactic acidosis, loss of cellular function and neuronal cell death. After resuscitation, there is replenishment of ATP and baby enters into a latent phase characterised by cerebral hypo perfusion with decreased metabolism, initiation of increased intracellular $\mathrm{Ca}^{2+}$ entry with glutamate excitotoxicity and reduced electroencephalogram (EEG)/amplitude integrated electroencephalogram (aEEG) activity. This latent phase continues for 6-15 hours and is followed by secondary energy failure. Latent phase is an important phase for application of neuroprotective strategies like therapeutic hypothermia (HT). Secondary energy failure is characterised by $\mathrm{Ca}^{2+}$ influx, glutamate excitotoxicity, secondary cytotoxic oedema, mitochondrial failure, reactive oxygen species (ROS) injury, cerebral hyper perfusion and cellular loss by apoptosis [5].

All resuscitated new-born babies should be assessed in the immediate post- natal life for features of HIE with Sarnat and Sarnat staging into mild, moderate and severe. The moderate and severe cases of HIE in term infants carry the risk of neonatal death and neurological sequelae [6].

\section{HIE staging}

- $\quad$ Stage 1 HIE (Sarnat and Sarnat) [7]: Is characterised by hyperirritability, hyper excitability, loss of sleep, sympathetic hyperactivity, lasts for a day and with excellent neurological outcome.

- $\quad$ Stage 2: Is characterised by depressed sensorium, hypotonia, hyporeflexia, parasympathetic hyperactivity and seizures.

- $\quad$ Stage 3: Is characterised by coma, no responsiveness, limp, reduced respiratory drive, areflexia, absent deep tendon reflexes, abnormal EEG and grave neurological outcome.

Thompson scoring system for HIE [8]

It is a clinical scoring system with 9 parameter signs namely muscle tone, level of consciousness, seizures, body posture, Moro reflex, grasp reflex, sucking reflex, respiratory pattern and tension of anterior fontanelle and scored 0 to 3 . Reflexes, seizures and fontanelle do not carry score three. Scoring should be recorded daily for prognostication.

\section{Prediction of HIE in post-resuscitated neonates}

Unless we identify the HIE of moderate to severe grade within the window period of 6 hours (preferably within 3hours for better motor outcomes, [9] after primary hypoxic-ischemic insult, neuroprotective and supportive measures cannot be initiated or referral to cooling centre is possible. Hence, it is crucial in detecting early HIE cases by cost effective clinical methods within 6hours.

Both one minute and five-minute Apgar scores of $<6$ and neonatal resuscitation with bag-valve-mask ventilation at birth (White., et al. 2012) are reliable early predictors of moderate to severe HIE. Raised Troponin $\mathrm{T}$ and blood lactate are simple biochemical spot tests in predicting moderate to severe HIE [10].

\section{Cooling criteria [11]}

All eligible term infants and late preterm infants with HIE stages 2 and 3 (preterm infants > 36 weeks GA and/ > 1800 gm birth weight) should be protected with therapeutic hypothermia (core temperature $33.5^{\circ} \mathrm{C}$ ) within six hours after primary insult for reducing mortality and neurological sequelae. The following entry criteria used for HT [11]. 
- $\quad$ Gestational age $>36$ weeks with $<6$ hours of postnatal age

- $\quad$ Criteria A: Suggests severe metabolic acidosis with at least one of the following - 1) Poor Apgar scores $<5$ at 10 minutes, 2) Cord or postnatal blood $\mathrm{pH}<7$ or base excess (BE) $>-16 \mathrm{mmol} / \mathrm{L}$ within one hour of life, 3) Prolonged resuscitation for 10 minutes.

- Criteria B: It is clinical criteria with altered sensorium and at least one of the following - 1) abnormal muscle tone, 2) abnormal reflexes (including primitive and autonomic reflexes), 3) poor or absent suck.

- Criteria C: Clinical seizures or abnormal EEG/aEEG pattern.

HIE and therapeutic hypothermia in high income countries $[12,13]$

[12] in a systematic review with 11 randomized control trial (RCT) trials which included 1505 participants (term and late preterm infants) with moderate to severe HIE. The review showed that the HT was beneficial to HIE infants with moderate to severe grade with favourable outcomes at 18 months of age - death and major disability (combined death and major disability - RR 0.75, 95\% CI 0.68 - 0.83; and RD -0.15, 95\% CI 0.20 - 0.10; NNTB 7).

Shah PS., et al. 2015 [13] in a meta-analysis of 13 clinical trials showed that HT is a trusted treatment for HIE with definitive decrease in binary outcomes of mortality and neuro developmental disability in childhood (HT $n=800,=$ HT 479, RR 0.74, 95\%CI 0.65 - 0.83 , Heterogeneity $p=0.07$, test of oral effect $p=<0.0001$ ).

All HIE babies with moderate to severe grade and more than $>36$ weeks GA with chronological age of $<6$ hours and fitting into criteria $A, B$ and $C$ should be treated with therapeutic hypothermia if available.

\section{HIE and cooling in low to middle income countries}

Most of the global neonatal deaths due to perinatal asphyxia are occurring in low to middle income countries (LMIC). These eligible babies are not treated with HT due to various reasons prevailing in these countries like [14]

- $\quad$ Paucity of tertiary neonatal intensive care centres equipped with men and machine.

- High-cost neonatal equipment for HT and CFM (cerebral function monitor).
- High incidence of home deliveries especially in rural areas with poor neonatal transport system. Precious time of early six hours of life is lapsed at primary care centres and during transport.

- High incidence of early onset neonatal sepsis (EOS) and intrauterine growth retardation (IUGR). In a recent study, [15] showed the safety and efficacy of HT in infants with HIE and EOS.

- Inclusion criteria for HT is not applicable in following circumstances like preterm and low birth weight HIE babies, congenital anomalies, late NICU admissions after six hours of life, mild HIE and life-threatening cardiorespiratory disorders.

In a meta-analysis, [14] showed that the HT in LMIC countries was not significantly lowered the primary outcome (mortality). Of 567 infants in seven RCTs, four studies were whole body cooling and three studies with selective head cooling. There were 15\% mild, $48 \%$ moderate HIE cases and did not ventilate in $88 \%$ infants. Various indigenous simple cooling devices used for HT without statistical benefit (RR 0.74, 95\% CI: 0.44 to 1.25). This could be because of poor efficacy of cooling devices.

A recent large multi-centric, multinational [16] in Southeast Asia showed that therapeutic hypothermia (servo control) in infants with neonatal encephalopathy in LMIC is not beneficial and more over showed increased neonatal mortality. Thus, this study suggested not to offer HT as standard care treatment for HIE infants in LMIC but needs more studies before recommendation.

\section{HIE and therapeutic strategies at low resource setting}

In a recent systematic review by [10] showed pooled evidence for three components of HIE namely 1 . Prediction of HIE in resuscitated neonates at birth 2. Monitoring of HIE and 3. Potential management strategies for HIE at low resource setting. In a clinical situation where HT is neither available nor in time referral to cooling centre possible as in low to middle income countries (LMIC), the potential management strategies include to ensure normothermia/passive cooling/prevention of hyperthermia and prevention of secondary brain injury and multi organ dysfunction by ensuring cardio pulmonary stability, prophylactic early theophylline to prevent renal dysfunction, avoiding fluid restriction, adequate perfusion of brain and other organs, intravenous magnesium sulphate 
infusion, prophylactic phenobarbitone for prevention and early management of seizures, low dose erythropoietin and maintaining euglycemia. Therapeutic hypothermia is possible in LMIC countries with Phase Changing Material (Meera Cradle in India).

Cooling hie cases who do not fit into cooling criteria

[17] showed that cooling the new-borns that were excluded by inclusion criteria for HT, had better outcomes - both short and long term. Better outcomes were noticed in all study subgroups except in infants with major intra cranial haemorrhage. The percentage of poor outcomes among the subgroups were - cooled late 31\%, postnatal collapse $-38 \%$, preterm ( $<36 \mathrm{wk}$ GA)-25\%, major ICH$80 \%$, cardiac case- $50 \%$, surgical case-50\% and with fulfilled entry critera-45\%. Poor outcome was defined as either neonatal death or MDI/PDI $<70$.

Other potential neuroprotective strategies for HIE

These strategies include allopurinol therapy, stem cell therapy and xenon inhalation with varying success.

- Allopurinol: Is a xanthine oxidase inhibitor. It is tried in reducing xanthine oxidase mediated free radical neuronal injury in HIE infants. In a meta-analysis (Chaudhari T., et al. 2012 -Cochrane database of systematic review, CDSR), Allopurinol did not show statistical benefit for death (RR 0.88; 95\% CI 0.56-1.38; risk difference -0.04; 95\% CI -0.18-0.10) and combined death and major disability (RR 0.78; 95\% CI 0.56-1.08; risk difference -0.14 ; 95\% CI -0.31-0.04) [18].

- Stem cell-based therapies: There is a potential role for stem cell-based therapies in HIE to repair or replace the injured neuronal cells. In a Cochrane review with three phase1, open label trials showed neither benefit nor harm of these therapies in preventing the binary outcomes of death and disability in HIE infants [19].

- XENON: Is a NMDA receptor antagonist and neuro-protective in neonatal rats with HIE. It has been tried as an adjuvant therapy to HT in HIE infants. In a CDSR review with 1 RCT including 92 neonates were treated with $30 \%$ Xenon as an adjuvant therapy to HT. The study did not show beneficial effects in HIE and hence, not recommended for routine use in HIE infants. Xenon is a costly drug [20].

\section{Outcome predictors after HIE}

Predictors help in recognising the high-risk neonates for poor outcomes. Poor outcomes after HIE are defined as death, developmental quotient- DQ $<70$ and severe cerebral palsy (CP).
Mnemonic: A: Apgar scores at 10 minutes, B: Biochemistry, C: Convulsions D: Doppler findings of major cerebral arteries, E: Examination (neurological), F: Functional Monitoring of cerebrum (CFM - cerebral functional monitoring), G: grading of HIE, H: pH of cord blood, I: Imaging (MRI, MR spectroscopy and cUSS).

- Apgar scores: Less than $<4$ at 10 minutes is associated with poor outcome in hypothermic babies [21]. In a study, zero percent disability free survived infants were found with zero apgar scores at 10 minutes [22].

- Biochemical tests: Blood Lactate is a poor predictor of outcome as a single biomarker but its predictivity increases (> $4.4 \mathrm{mmol} / \mathrm{L}$ ) in combination with creatinine kinase CK, lactate dehydrogenase $\mathrm{LDH}$ and uric acid [23,24]. Cord $\mathrm{pH}<7$ and $\mathrm{BE}>-12 \mathrm{mmol} / \mathrm{L}$ are early signs of perinatal asphyxia and carry abnormal outcome. The PPV is increased if serum lactate $(>4.4 \mathrm{mmol} / \mathrm{L})$ has been added. The BE $>-6.2$ in $<4 \mathrm{hr}$ after birth carry bad outcome in normothermic babies [14]. Base deficit of more than 22 during first day of life predicts death [22]. Cardiac Troponin I level $<0.15 \mathrm{ng} / \mathrm{ml}$ at 24 hours in cooled term infants carry good prognosis and increased levels indicate cardiac myocytes injury [25]. LDH - increased levels indicate multi organ injury. LDH levels > $1050 \mathrm{IU} / \mathrm{L}$ in less than 12 hours predicts NE in term infants. Its levels < $2085 \mathrm{IU} / \mathrm{L}$ predicts good outcome and if more, predicts 55\% poor outcome. Levels > 5090 IU/L and > 3555 IU/L were associated with death and disability in cooled babies respectively [24].

- Convulsions/seizures: Infants with multifocal seizures, status epilepticus and seizures requiring multiple anti-epileptic drugs were associated with more severe changes on MRI brain scan and its associated bad outcomes [26].

- Doppler findings - Cranial ultrasound scan (cUSS): Early deep gray matter injury carries poor outcome and death. Doppler resistance index (RI) of $<0.55$ in major cerebral arteries within 24-62 hours in normothermic infants carries bad outcome with PPV 75\% and NPV 100\% [27]. Recovering RI values of $>0.55$ in hypothermic babies after rewarming carries good outcome [28].

- Examination findings (Neurological): Neurological assessment: Inability to suck and swallow at discharge carries poor outcome at 18 months of age [29]. 
- Functional monitoring of cerebrum (CFM): EEG/aEEG: Early abnormal EEG in $<6$ hours is the best predictor of future outcome in normothermic HIE babies [30]. Restoration of normal aEEG background activity by 48 hours predicts good outcome in hypothermic babies [31]. Infants who don't regain normal aEEG at any time carry $70 \%$ mortality/bad outcome. Seizures in $<4$ hours carry bad prognosis. Restoration of sleep wake cycle $<36 \mathrm{hr}$ in normothermia babies carry good outcome.

- Grading of HIE: Sarnat and Sarnat HIE stage 2 and 3 carry poor outcomes in both cooled and non-cooled infants. Thompson score of $>16$ in first 6 hrs carries bad outcome. A score less than 10 at admission and reaching 0 by one week carries good prognosis [8]. A score of more than 10 on day 3 and $>7$ on day 10 carry bad outcome with PPV 73\%, NPV 94\% and PPV 63\%, NPV 100\% respectively in non-cooled babies. Scores on day 7 , if found $<5$ or $>7$ carry good or bad outcome respectively [32]. H. Blood pH: Already mentioned above.

Imaging: Magnetic resonance imaging (MRI):

MRI gives timing, pattern and extent of brain injury with prognostic value in both hypothermic and normothermic HIE infants. Deep gray matter injury seen in profound HIE cases with associated bad outcome - death or disability (spastic quadriplegia/dyskinetic CP) and cognitive impairment -MDI < 70 [33]. PLIC (Posterior limb of internal capsule) sign is absence of its normal appearance in MRI imaging in term infants and carries poor outcome [34]. MR Spectroscopy (MRS) - An increase of lactate/NAA (N-acetyl aspartate) ratio highly predicts bad outcome in early infancy [35].

Cerebral near infrared spectroscopy

This advanced facility is not available in many LMIC countries. Volume weighted haemoglobin - oxygen saturation (StO2) is significantly higher in HIE infants (without therapeutic hypothermia) with poor outcomes [36].

\section{Predictors of good prognosis}

Normal EEG pattern at 3hours of life, aEEG showing sleep-wake pattern, background normalization of aEEG, increased RI values > 0.55 of major cerebral arteries on rewarming in HT babies, Thompson's score of $<5$ on day 7 and $<10$ at admission and reaching 0 by day7 on non-HT babies carry good prognosis.

\section{Futility prediction in HIE infants}

Consider taking multiple factors for futility prediction.

The dying HIE infants may have the following commons:

- Lower Apgar scores $<3$ at 10 minutes, cord $\mathrm{pH}<6.8$ and adrenaline usage.

- $\quad$ More oxygen requirement on day 1.

- Higher lactate levels in early hours $(<7 \mathrm{hr})$.

- Evidence of multiorgan dysfunction in $<24 \mathrm{hr}$.

- Abnormal aEEG and MRI patterns at 24 hours.

- No spontaneous activity.

- $\quad$ Fourfold increase in mortality or severe disability is associated with every $1^{\circ} \mathrm{C}$ rise in body temperature from $36.5^{\circ} \mathrm{C}$.

Counselling - discussion with parents regarding hie and outcome prediction

Hypoxic Ischaemic Encephalopathy is a devastating condition in newborn, hence discussion with parents should be carried in a soft and sympathetic way for the existing situation in counselling room. Explain the parents and family members with pictorial play charts regarding HIE and its causation, predictive biomarkers and outcome data. Reveal to parents the clinical diagnosis, HIE, its cause, evidence in support, present clinical status, early stabilisation measures, specific therapy - HT with its benefits and side effects, daily update, predictors of long term (poor) neuro-developmental outcomes. Discuss about length of hospital stay, financial issues, insurance, need for long term follow up and multidisciplinary rehabilitation services.

Take into account the emotional landscapes of parents [37], need for continuous support from doctors and nurses and to prepare the parents for reconciling the uncertain outcomes (Thyagarajan B 2018., et al.) [38].

\section{Follow up screening for neurodevelopmental outcome}

All HIE neonates should be followed up for screening - eye vision and hearing who were treated with or without HT especially when on aminoglycosides with Bayley's 3 or 4 developmental screen. A score of MDI OR PDI > 70 is considered normal. A score of $50-<70$ is considered moderate delay and $<50$ is considered as severe developmental delay. 
Long-term health problems

Approximately 50 million under-five children are experiencing developmental disabilities in LMIC countries and majority of whom $(80 \%)$ were contributed by perinatal cause. Children with severe neuro-developmental disability after HIE are at increased tendency to die and develop cerebral palsy, suffer from pain, memory impairment, loss of abstract thinking, earning potential, recreation and pleasures in life [39].

- Nutritional and Gastro-intestinal problems: Are common and include feeding difficulties, growth impairment, micro and macro nutritional deficiencies and aspiration pneumonias.

- Musculoskeletal problems: Generalised osteoporosis, kypho-scoliosis, hip dislocation, contractures, spasticity, rigidity, hypotonia, callosities at pressure sites.

- Cognitive Impairment: These children experience learning difficulties, low IQs, visual impairment, hearing impairment - sensory neural deafness and need special educational support.

- Behavioural Problems: Include attention deficit, explosiveness and hyperirritability.

\section{Conclusion}

HIE infants are great burden to health care sector in LMIC countries. Therapies that are in use in NICUs of advanced countries are not practical in LMIC countries. Treating these babies is still a challenging task in LMIC countries. Cost effective strategies can be applied to treat these babies at low resource setting like optimum neonatal resuscitation in experienced hands, prevention of hyperthermia, controlled passive cooling, maintaining euglycemic state by prompt recognition and management of hypo - hyper glycaemia, avoiding hyperoxia and hypocarbia, early erythropoietin therapy, early prophylactic theophylline therapy, avoiding fluid restriction, early phenobarbitone for prevention of seizures, cardiopulmonary stabilization and early therapeutic hypothermia preferably within 3hours with phase changing material.

\section{Conflict of Interest}

There are no conflicts of interest for this study.

\section{Sponsors}

There are no sponsors for this study.
Bibliography

1. Lee ACC., et al. "Intrapartum - related neonatal encephalopathy incidence and impairment at regional and global levels for 2010 with trends from 1990". Pediatric Research 74.S1 (2013): 50-72.

2. Montaldo P., et al. "Cooling in a low-resource environment: Lost in translation". Seminars in Fetal and Neonatal Medicine (2014): 1-8.

3. Basic New-born Resuscitation: A Practical Guide,.4, Geneva: World Health Organization; (1997).

4. "Neonatal Encephalopathy and Neurologic Outcomes. Second Edition" American Congress of Obstetrics and Gynaecology 123.4 (2014).

5. Drury PP., et al. "Mechanisms of hypothermic neuro-protection". Seminars in Fetal and Neonatal Medicine 15.5 (2010): 287-292.

6. pe JJ. "Neurology of Newborn, 5th Edition".

7. Sarnat HB and Sarnat MS. "Neonatal Encephalopathy Following Fetal Distress - A Clinical and Electroencephalographic Study". Archives of Neurology 33 (1976).

8. Thompson CM., et al. "The value of a scoring system for hypixic ischemic encephalopathy in predicting neurodevelopmental outcome". Acta Paediatrics 86 (1997): 757-761.

9. Thoresen M., et al. "Time is brain: starting therapeutic hypothermia within 3hours after birth improves motor outcomes in asphyxiated newborns". Neonatology 104 (2013): 228-233.

10. Maddela C., et al. "Enhanced Post-resuscitation Neonatal Care in Possibly Hypoxic Neonates at Resource Limited Setting: A Systematic Review". Acta Scientific Paediatrics 4.7 (2021): 7786.

11. Azzopardi., et al. Toby Study Group - "Moderate hypothermia to treat perinatal asphyxial encephalopathy". The New England Journal of Medicine 361.14 (2009): 1349-1358.

12. Jacobs SE., et al. "Cooling for new-borns with hypoxic-ischaemic encephalopathy". Cochrane Database Systematic Reviews 2013 (2013): CD 003311.

13. Shah PS., et al. "Hypothermia: a systematic review and metaanalysis of clinical trials". Seminars in Fetal and Neonatal Medicine 15 (2010): 238-246. 
14. Pauliah SS., et al. "Therapeutic Hypothermia for Neonatal Encephalopathy in Low- and Middle -Income countries: A Systematic Review and Meta-Analysis". PLOS ONE 8.3 (2013).

15. Marian H., et al. "Outcome of Infants with Therapeutic Hypothermia after Perinatal Asphyxia and Early-Onset Sepsis". Neonatology 115.2 (2018): 127-133.

16. Thayyil S., et al. "Hypothermia for moderate to severe neonatal encephalopathy in low-income and middle-income countries (HELIX): a randomized controlled trial in India, Srilanka and Bangladesh". Lancet 9.9 (2021): e1273-e1285.

17. Smit E., et al. "Cooling neonates who do not fulfil the standard cooling criteria - Short-and Longterm outcomes". Acta Paediatrica 104.2 (2014): 138-145.

18. Chaudhari T and McGuire W. "Allopurinol for preventing mortality and morbidity in newborn infants with Hypoxic Ischemic Encephalopathy". CDSR (2012).

19. Bruschettini M., et al. "Stem cell-based interventions for the prevention of morbidity and mortality following Hypoxic Ischemic Encephalopathy in new-born infants CDSR Interventional review". Cochrane Database of Systematic Reviews 8.8 (2020): CD013202.

20. Ruegger CM., et al. "Xenon as an adjuvant to therapeutic hypothermia in near-term and term newborns with hypoxicischemic encephalopathy". Cochrane Database of Systematic Reviews Interventional Review 8.8 (2018): CD012753.

21. Laptook AR., et al. "Outcome of term infants using apgar scores at 10 minutes following hypixic - ischemic encephalopathy". Pediatrics 124 (2009): 1619-1626.

22. Sarkar., et al. "Predicting death despite therapeutic hypothermia in infants with HIE". Archives of Disease in Childhood - Fetal and Neonatal Edition 95 (2015): 423-428.

23. Shah S., et al. "Postnatal lactate as an early predictor of shortterm outcome after intrapartum asphyxia". Journal of Perinatology 24 (2004): 16-20.

24. Sabir H., et al. "Prediction of outcome methods assessing short-and long-term outcome after therapeutic hypothermia". Seminars in Fetal and Neonatal Medicine 20 (2015): 115-121.

25. Liu X., et al. "Effect of cardiac compressions and hypothermia treatment on cardiac troponin I in newborns with perinatal asphyxia". Resuscitation 84.11 (2013): P1562-1567.
26. Shah DK., et al. "Electrographic seizures are associated with brain injury in newborns undergoing therapeutic hypothermia". ADC Fetal and Neonatal 99.3 (2014): F219-224.

27. Eken P., et al. "Predictive value of early neuroimaging, pulsed Doppler and neurophysiology in fullterm infants with hypoxic-ischemic encephalopathy". Archives of Disease in Childhood. Fetal and Neonatal Edition 73 (1995): F75-F80.

28. Skranes JH., et al. "Hypothermia makes cerebral resistance index a poor prognostic tool in encephalopathic newborns". Neurology 106 (2014): 17-23.

29. Shankaran S., et al. Eution of encephalopathy during "Whole body hypothermia for neonatal hypoxic-ischemic encephalopathy". The Journal of Pediatrics 160 (2012): 567-572e3.

30. Ellstrom-Westas L., et al. "Predictive value of early continuous amplitude integrated EEG recordings on outcome after severe birth asphyxia in fullterm infants". Archives of Disease in Childhood. Fetal and Neonatal Edition 72 (1995): F34-F38.

31. Thoresen M., et al. "Effect of hypothermia on amplitude-integrated electroencephalogram in infants with asphyxia". Pediatrics 126 (2010): e131-e139.

32. Simbruner G., et al. "Systemic hypothermia after neonatal encephalopathy: outcomes after neo. nEURO. network RCT". Pediatrics 126 (2010): e771-e778.

33. Bednarek N., et al. "Impact of therapeutic hypothermia on MRI diffusion changes in Neonatal Encephalopathy". Neurology 78.18 (2012): 1420-1427.

34. Rutherford MA., et al. "Abnormal magnetic resonance signal in the internal capsule predicts poor neurodevelopmental outcome in infants with hypoxicischaemic encephalopathy". Pediatrics 102.2Pt 1 (1998): 323-328.

35. Barkovich AJ., et al. "MR Imaging, MR Spectroscopy, and Diffusion Tensor Imaging of Sequential Studies in Neonates with Encephalopathy". AJNR 27 (2006): 533-547.

36. Toet MC., et al. "Cerebral oxygenation and electrical activity after birth asphyxia: their relation to outcome". Pediatrics 117 (2006): 333-339.

37. Nassef SK., et al. "Experiences of Parents Whose Newborns Undergo Hypothermia Treatment Following Perinatal Asphyxia". JOGNN 42 (2013): 38-47. 
38. Thyagarajan B., et al. "Parental perceptions of hypothermia treatment for neonatal hypoxicischemic encephalopathy". The Journal of Maternal - Fetal and Neonatal Medicine 31.19 (2018): 2527-2533.

39. Eunson P., et al. "Long-term health, social and financial burden of Hypoxia-Ischemia Encephalopathy". Developmental Medicine and Child Neurology, HIE supplement 57.3 (2005): 48-50.

\section{Assets from publication with us}

- Prompt Acknowledgement after receiving the article

- Thorough Double blinded peer review

- Rapid Publication

- Issue of Publication Certificate

- High visibility of your Published work

Website: www.actascientific.com/

Submit Article: www.actascientific.com/submission.php

Email us: editor@actascientific.com

Contact us: +919182824667 